\title{
REDUCTION OF THE INTERACTION BETWEEN VITRONECTIN AND CARDIAC CUSHION MESENCHYMAL CELLS OF THE RAT EMBRYO BY BISDIAMINE
}

\author{
Hiroshi Sumida ${ }^{1,2}$, Harukazu Nakamura ${ }^{3}$, Miki Hisakane ${ }^{1}$ and Mineo Yasuda ${ }^{2}$ \\ ${ }^{1}$ Anatomy and Physiology Division, Sanyo Women's College, Sagatahonmachi, Hatsukaichi, Hiroshima 738, ${ }^{2}$ Department of \\ Anatomy, Hiroshima University School of Medicine, Kasumi, Minamiku, Hiroshima 734, and ${ }^{3}$ Department of Biology, Kyoto \\ Prefectural University of Medicine, Nishitakatsukasacho, Taishogun, Kitaku, Kyoto 603, Japan
}

\begin{abstract}
A teratogen, $N, N^{\prime}$-bis-(dichloroacetyl)-diamine,1,8,-octa methylene diamine (bisdiamine) induces endocardial cushion defects in the rat. We examined the effect of bisdiamine on the interaction between embryonic endocardium-derived cushion mesenchymal cells and extracellular matrix. Bisdiamine suspended in aqueous solution of gum tragacanth was orally administered to maternal rats at a dose of $200 \mathrm{mg} /$ day on days 9 and 10 of gestation. Cushion mesenchymal cells from 13-day-old embryos were cultured on vitronectin, fibronectin, collagen type I with a serum-free medium, and the migration distance of cushion mesenchymal cells was measured. On vitronectin, the migration distance of cushion mesenchymal cells of the group treated with bisdiamine was significantly shorter than that without the treatment. In contrast, on fibronectin, the migration distance of cushion mesenchymal cells of the group treated with bisdiamine was larger than that of the non-treated group. On vitronectin or fibronectin, the migration distance of cushion mesenchymal cells of the group treated with only gum tragacanth was not significantly different from that of the non-treated group. In the previous in vitro study, vitronectin was suggested to inhibit the migration stimulation of fibronectin. The results of the present study clearly show that the reaction of embryonic cushion mesenchymal cells against vitronectin was reduced in the group treated with bisdiamine. Such reduction of the interaction between cushion mesenchymal cells and vitronectin probably causes abnormal endocardial cushion formation.
\end{abstract}

During the cardiogenesis, swellings develop inside of the heart tube, and contribute to the morphogenesis of certain internal structures of the heart. The endocardial cushion, which develops in the atrioventricular canal, is one of the cardiac swellings, and is involved in the development of the atrioventricular septum. Mesenchymal cells in the endocardial cushion (cushion mesenchymal cells) are derived from the endocardium $(2,5,10,11)$. These cushion mesenchymal cells migrate toward the myocardium after being separated from the endocardial sheet. The migration of cushion mesenchymal cells is thought to be an important process for the endocardial cushion formation.

It has been shown that various extracellular matrices including fibronectin and collagen type I are involved in the migration of cushion mesenchymal cells $(1,3,6,7,9)$. Recently, we reported transient distribution of vitronectin in the cushion tissue during a period of the migration of cushion mesenchymal cells (14). In our in vitro study, vitronectin was suggested to play an important role for attachment of cushion mesenchymal cells to the substratum (15).

Oral administration of $N, N^{\prime}$-bis-(dichloroacetyl)diamine, 1,8-octa methylene diamine (bisdiamine) to the pregnant rat induces endocardial cushion defects in siblings $(12,13,16)$. Growth retardation and malposition of the cardiac swellings were observed in the embryos from mothers treated with 

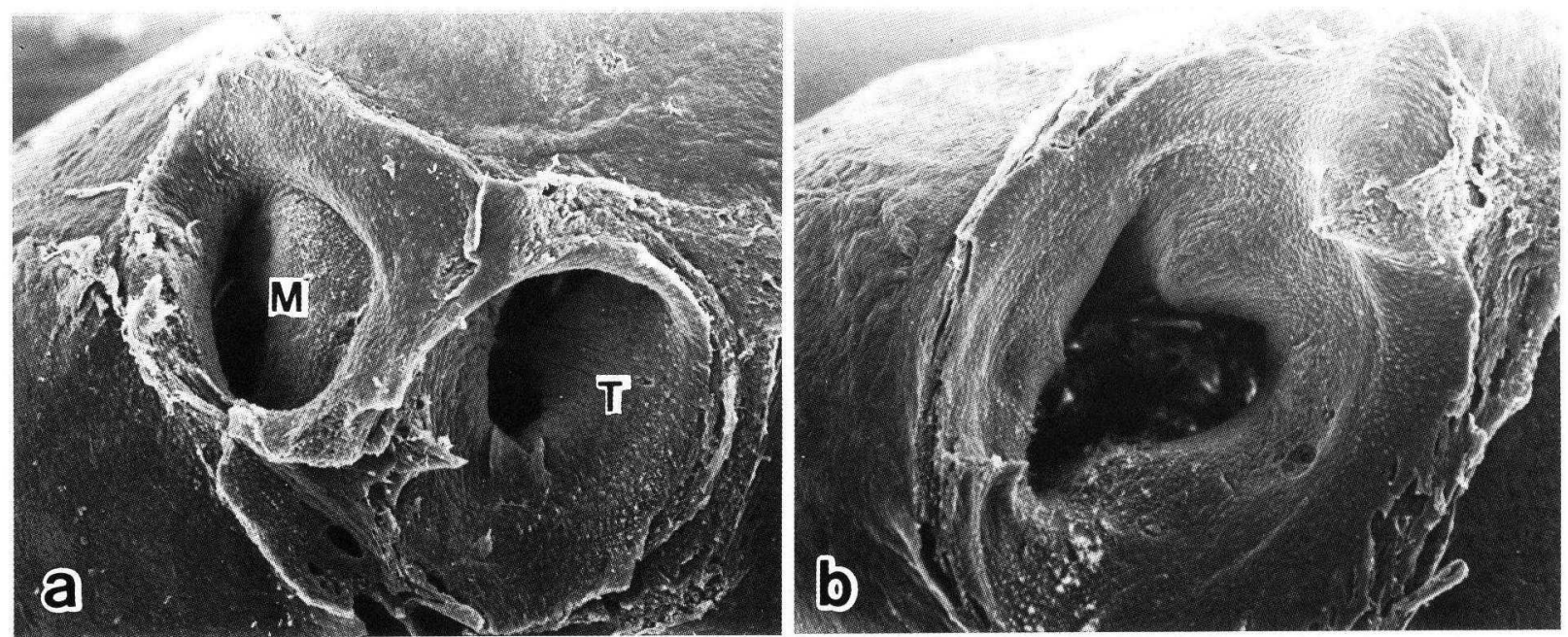

Fig. 1 Scanning electron micrographs of the atrioventricular valve of a non-treated control embryo (a) and an embryo from a mother treated with bisdiamine (b). The mitral $(\mathrm{M})$ and tricuspid $(\mathrm{T})$ valves are seen in the control embryo (a), but the atrioventricular orifice persists in the embryo from the group treated with bisdiamine (b).

bisdiamine (12). Precise pathogenesis of maldevelopment of the endocardial cushion by the maternal treatment of bisdiamine are still unclear. It has been suggested that bisdiamine affects the migration of the neural crest cells (13). From this point of view, migration of cushion mesenchymal cells may be disturbed by bisdiamine. However, decay of migration ability of cushion mesenchymal cells has not been demonstrated.

This study was performed to know whether bisdiamine interferes with interaction between cushion mesenchymal cells and extracellular matrix. For this purpose, we examined the response of cushion mesenchymal cells of embryos from the mother treated with bisdiamine to the migration stimulation of vitronectin, fibronectin and collagen type I.

\section{MATERIALS AND METHODS}

Donryu strain rats were used. Day 0 of gestation was assigned by the appearance of sperms in a vaginal smear. On both days 9 and 10 of gestation, $200 \mathrm{mg}$ of bisdiamine (Sigma, U.S.A.) suspended in $1 \mathrm{ml}$ of $1 \%$ aqueous solution of gum tragacanth was administered orally. Administration at this period of gestation induced endocardial cushion defects in embryos (Fig. 1). Non-treated rats were used as controls. In order to examine the effect of gum tragacanth to the migration distance of cushion mesenchymal cells on vitronectin or fibronectin, $1 \mathrm{ml}$ of $1 \%$ aqueous solution of gum traga- canth was administered orally on both days 9 and 10 of gestation. This was used as gum tragacanth treated controls. Rats were sacrificed on day 13 of gestation, and embryos (E13 embryos) were removed.

\section{Cell Migration Assay}

Tissue culture dishes $(3.5 \mathrm{~cm}$ diameter, Becton Dickinson Microbiology System, Cockeysville, U.S.A.) were coated with $15 \mu \mathrm{g}$ of vitronectin or fibronectin, or $150 \mu \mathrm{g}$ of collagen type I (Nitta Gelatin, Yao, Japan). As described in a previous paper (15), vitronectin and fibronectin were purified from human plasma by methods by Yatohgo et al. (17) and Hayashi et al. (4), respectively.

The endocardial cushion of E13 embryos was cut off under a stereomicroscope, and was cultured on the dishes prepared as mentioned above with Dulbecco's modified Eagle's medium (Nissui Pharmaceutical, Tokyo, Japan) including $2 \mathrm{mM} \mathrm{L-gluta-}$ mine, $100 \mathrm{U} / \mathrm{ml}$ penicillin, $100 \mu \mathrm{g} / \mathrm{ml}$ streptomycin sulfate and $0.25 \mu \mathrm{g} / \mathrm{ml}$ fungizon (Irvine Scientific, Santa Ana, U.S.A.) at $38^{\circ} \mathrm{C}$ with humidified air containing $5 \% \mathrm{CO}_{2}$. Number of tissue explants was shown in Table 1.

After $48 \mathrm{~h}$ of incubation, the culture was fixed with $4 \%$ paraformaldehyde in $0.1 \mathrm{M}$ phosphate buffer ( $\mathrm{pH} 7.4$ ) for $4 \mathrm{~h}$. The explanted tissues and cushion mesenchymal cells which migrated out from the explant were photographed. The migra- 
Table 1 Number of Explants Cultured on Vitronectin (VN), Fibronectin (FN), Collagen Type I (CI) or Non-Substratum-Coated Plastic $(N O N)$

\begin{tabular}{cccc} 
Substratum & $\begin{array}{c}\text { Bisdiamine } \\
\text { treatment }\end{array}$ & Control & $\begin{array}{c}\text { Gum tragacanth } \\
\text { treatment }\end{array}$ \\
\hline VN & 44 & 26 & 24 \\
FN & 37 & 31 & 17 \\
CI & 23 & 23 & - \\
NON & 8 & 10 & - \\
\hline
\end{tabular}

tion distance was measured on the micrograph as described in the previous paper (15).

\section{Immunocytochemistry}

Immunocytochemical procedure was performed at room temperature. After being photographed, the cells were washed with phosphate-buffered saline (PBS) for $1 \mathrm{~h}$. The cells were incubated with the anti-vitronectin antibody for $2 \mathrm{~h}$ (15). After washing with PBS for $15 \mathrm{~min}$, cells were incubated with the FITC-labeled anti-rabbit IgG (Zymed Labs, South San Francisco, U.S.A.) with normal goat serum for $1 \mathrm{~h}$. The specimens were mounted with glycerol/PBS (9:1) containing 10\% p-phenylenediamine to avoid fading of fluorescence (8).

\section{RESULTS}

On vitronectin or fibronectin, migration distance of cushion mesenchymal cells of the group treated with only gum tragacanth was slightly shorter than that of non-treated controls, however, no significant difference was revealed between them $(P>0.05)$. Hence, in most of the study, the nontreated group was used as the control.

In culture, shape of cushion mesenchymal cells was dependent on the substratum. On fibronectin, cells in a spindle shape were numerously observed (Fig. 2c). Cells in a cobble stone shape were spread on collagen type I (Fig. 2e). On fibronectin or collagen type I, morphological changes in the culture were not recognizable between the group treated with bisdiamine and non-treated controls (Fig. 2, d and f). On vitronectin, the cell size of cushion mesenchymal cells of the group treated with bisdiamine was slightly larger than that of non-treated controls (Fig. 2, a and b). Compared with nontreated controls, increase in the number of dead cells was not recognizable in the group treated with bisdiamine.
The migration distance of cushion mesenchymal cells was distinct on vitronectin, fibronectin, and collagen type I, respectively (Fig. 3). Bisdiamine affected the migration distance of cushion mesenchymal cells on vitronectin or fibronectin. On vitronectin, the migration distance of cushion mesenchymal cells of the group treated with bisdiamine was significantly decreased $(P<0.05)$ compared with that of non-treated controls or gum tragacanth treated controls (Fig. 3). In contrast, the migration distance of the group treated with bisdiamine was dramatically increased $(P<0.05)$ when cells were cultured on fibronectin. On collagen type I or non-coated plastic, there were no differences in migration between the group treated with bisdiamine and non-treated controls.

By immunocytochemistry, cushion mesenchymal cells of non-treated controls cultured on vitronectin were not so intensely stained with an antivitronectin antibody (Fig. 4a). Since vitronectin coated on the tissue culture dish reacted with the anti-vitronectin antibody, intense fluorescence was seen outside of cells. The similar feature was observed in the group treated with bisdiamine (Fig. 4b). Endogenous vitronectin was observed as a fibrous pattern of immunofluorescence in the cushion mesenchymal cells of non-treated controls cultured on fibronectin (Fig. 4c). In the cushion mesenchymal cells of the group treated with bisdiamine, similar fibrous pattern of fluorescence was observed. On collagen type I, intense fluorescence of anti-vitronectin staining was also recognizable in both non-treated controls (Fig. 4e) and the group treated with bisdiamine (Fig. 4f).

\section{DISCUSSION}

The present study showed 1) the migration distance of cushion mesenchymal cells of the group treated with bisdiamine was significantly shorter than that of non-treated controls or of gum traga- 

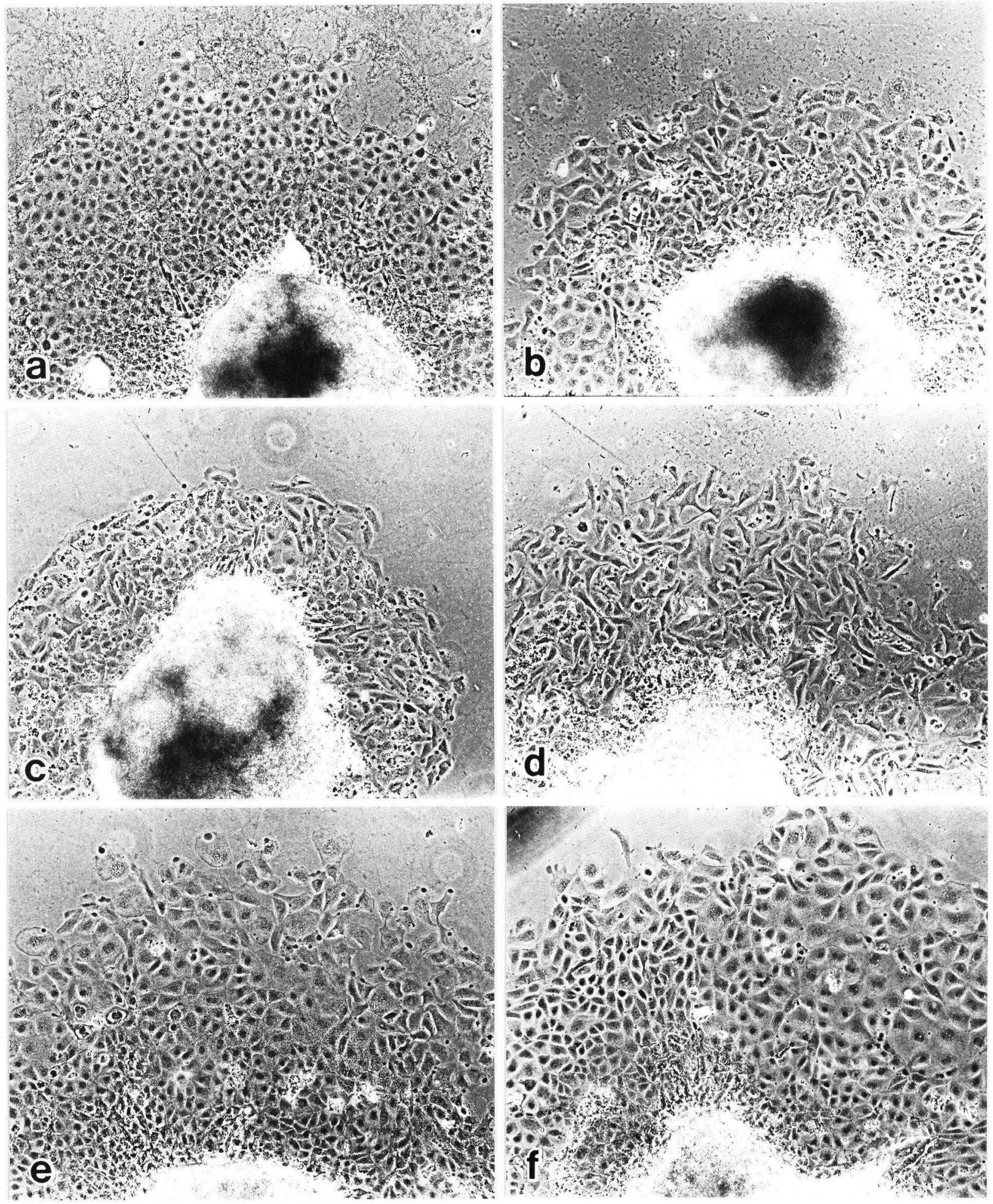

Fig. 2 Phase contrast micrographs of the culture of cushion mesenchymal cells of non-treated controls (a, c, e) or the group treated with bisdiamine $(b, d, f)$. The cell shape on the different substratum is distinctive (a, $b$ : on vitronectin, c, d: on fibronectin, e, f: on collagen type I). $\times 70$ 


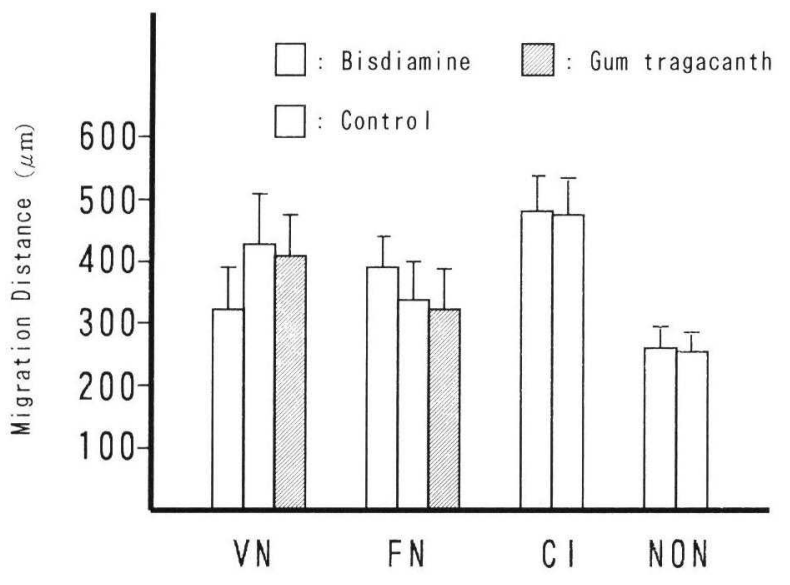

Fig. 3 Migration distance $( \pm S D)$ of cushion mesenchymal cells cultured on vitronectin (VN), fibronectin (FN), collagen type I (CI) or non-coated plastic (NON). On vitronectin, the migration distance of cushion mesenchymal cells from the group treated with bisdiamine is significantly shorter than that of non-treated controls or of gum tragacanth-treated controls. In contrast, on fibronectin, the migration distance of cushion mesenchymal cells of the group treated with bisdiamine is significantly larger than that of non-treated controls or of gum tragacanth-treated controls. The migration distance of cushion mesenchymal cells of the group treated with bisdiamine and non-treated controls is similar on collagen type I or non-coated plastic.

canth treated controls on vitronectin; in contrast, 2) on fibronectin, the migration distance of the cells of the group treated with bisdiamine was significantly larger than that of non-treated controls or of gum tragacanth treated controls. These results indicate that bisdiamine affected the migration ability of cushion mesenchymal cells.

When cushion mesenchymal cells were cultured on vitronectin, it promoted cell migration (15). Decrease in the migration distance of cushion mesenchymal cells of the treated group suggests that bisdiamine reduced the reaction of the cells to vitronectin.

On the other hand, endogenous vitronectin does not promote but perturbs cell migration when cushion mesenchymal cells were cultured on fibronectin. Fibronectin promotes rat cushion mesenchymal cell migration in vitro (15). Recently, Icardo et al. (7) demonstrated contribution of fibronectin in the separation of chick cushion mesenchymal cells from the endocardium in vivo. The previous (15) and the present study showed that rat cushion mesenchymal cells produce vitronectin or immu- nocytochemically vitronectin-like substance when the cells were cultured on fibronectin or collagen type I. In the previous study (15), we showed that endogenous vitronectin or the vitronectin-like substance perturbed the migration stimulation activity of fibronectin. Probably, cushion mesenchymal cells interact much stronger with vitronectin than with fibronectin, and endogenous vitronectin or the vitronectin-like substance cannot so widely spread on surface of the culture dish. Then, endogenous vitronectin or the vitronectin-like substance may be anchored the cells on fibronectin. Thus, increase in the migration distance of cushion mesenchymal cells of the group treated with bisdiamine also suggests that the reaction of the cells of the group treated with bisdiamine to vitronectin was diminished. Indeed, the migration distance of cushion mesenchymal cells on fibronectin was dramatically increased by blocking interaction between the cells and endogenous vitronectin or the vitronectin-like substance by addition of anti-vitronectin IgG in the culture medium (15).

Reduction of amount of endogenous vitronectin or the vitronectin-like substance possibly increase migration distance of cushion mesenchymal cells on fibronectin. In the present study, however, it is not clear whether production of vitronectin by cushion mesenchymal cells of the group treated with bisdiamine was reduced or not, because we did not carry out quantitative analysis.

In the culture, no damage of cushion mesenchymal cells of the group treated with bisdiamine could be observed compared with non-treated controls. This observation suggests elevation or degradation of migration distance of cushion mesenchymal cells of the group treated with bisdiamine is not based on morphological damage of the cells. Minor ultrastructural changes like vacuolation of the mitochondria and endoplasmic reticulum, however, might have been induced in the cells of the group treated with bisdiamine (12).

In this study, bisdiamine was administered to the maternal rats. Direct effects of bisdiamine to embryonic cells have not been elucidated. Because of water insolubility of bisdiamine, test of cell toxicity of bisdiamine in vitro is technically difficult.

In the developing heart of embryos from mothers treated with bisdiamine, the cardiac swellings were hypoplastic and malpositioned (12). No systematic study of the abnormality of the endocardial cushion of the bisdiamine treated group has been appeared. However, figures in the published study (Figs. 6-10 in reference 12) and unpublished obser- 

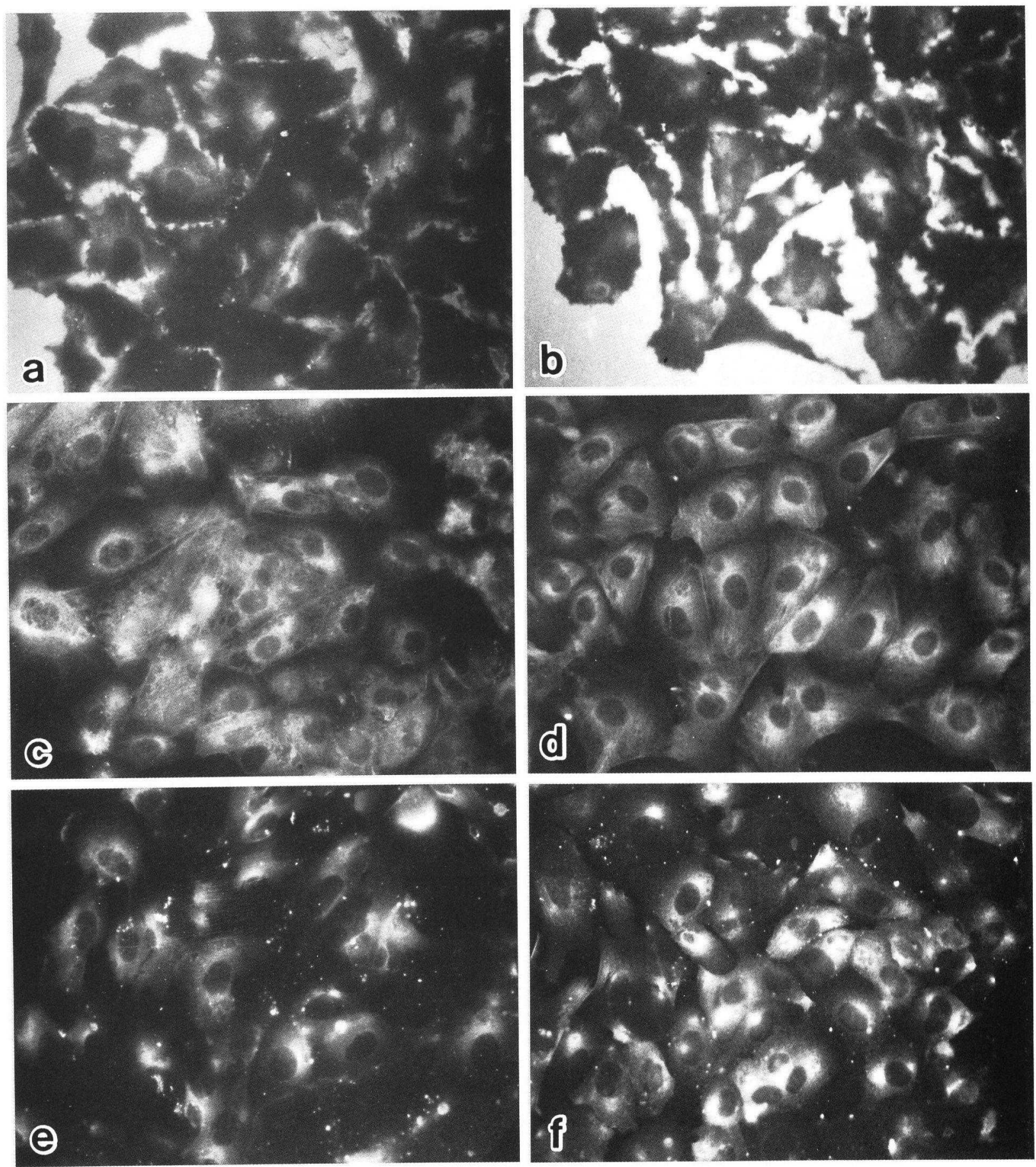

Fig. 4 Immunofluorescent micrographs of anti-vitronectin staining on cultured cushion mesenchymal cells of non-treated controls ( $a, c, e)$ or the group treated with bisdiamine (b, d, f). On vitronectin, weak staining of cells is seen in cushion mesenchymal cells of both group treated with bisdiamine and non-treated controls $(a, b)$. Note fluorescence by secondary antibody that bound vitronectin coated on the dish. Intense immunofluorescence is seen in cushion mesenchymal cells of non-treated controls on fibronectin (c) or collagen type I (e, f). $\times 500$ 
vation (Tasaka et al., personal communication) show that the endocardial cushion of the treated group was hypoplastic and malpositioned. Furthermore, Okishima et al. (13) suggested from their findings of persistent atrioventricular canal induced by bisdiamine, that bisdiamine might affect the growth of cushion mesenchymal cells. Contribution of vitronectin to the endocardial cushion formation in vivo is still unclear. The present study, however, clearly showed that the interaction between the cushion mesenchymal cells and vitronectin was disturbed by bisdiamine. This result may be the first demonstration at the cellular level effect of bisdiamine to cell migration, and suggests that vitronectin may play an important role for the endocardial cushion formation.

Received 24 April 1992; and accepted 30 June 1992

\section{REFERENCES}

1. Davis L. A., Ogle R. C. and Little C. D. (1989) Embryonic heart mesenchymal cell migration in laminin. Develop. Biol. 133, 37-43

2. Fitzharris T. P. and Markwald R. R. (1982) Cellular migration through the cardiac jelly matrix: A stereoanalysis by high-voltage electron microscopy. Develop. Biol. 92, 315-329

3. Funderburg F. M. and Markwald R. R. (1986) Conditioning of native substrates by chondroitin sulfate proteoglycans during cardiac mesenchymal cell migration. $J$. Cell Biol. 103, 2475-2487

4. Hayashi M. and Yamada K. M. (1982) Divalent cation modulation of fibronectin binding to heparin and to DNA. J. Biol. Chem. 257, 5263-5267

5. ICARDo J. M. (1989) Changes in endocardial cell morphology during development of the endocardial cushions. Anat. Embryol. 179, 443-448

6. ICARdo J. M. and ManASEK F. J. (1984) An indirect immunofluorescence study of the distribution of fibronectin dur- ing the formation of the cushion tissue mesenchyme in the embryonic heart. Develop. Biol. 101, 336-345

7. Icardo J. M., Nakamura A., Fernandez-Teran M. A. and MANASEK F. J. (1992) Effects of injecting fibronectin and antifibronectin antibodies on cushion mesenchyme formation in the chick: An in vivo study. Anat. Embryol. 185, 239-247

8. Johonson G. D., Nogueria Araujo G. M. De C. (1981) A simple method of reducing the fading of immunofluorescence during microscopy. J. Immunol. Meth. 43, 349-350

9. Loeber C. P. and Runyan R. B. (1990) A comparison of fibronectin, laminin, and galactosyltransferase adhesion mechanisms during embryonic cardiac mesenchymal cell migration in vitro. Develop. Biol. 140, 401-412

10. Markwald R. R., Fitzharris T. P. and Adams Smith W. N. (1975) Structural analysis of endocardial cytodifferentiation. Develop. Biol. 42, 160-180

11. Markwald R. R., Fitzharris T. P. and Manasek F. J. (1977) Structural development of endocardial cushions. Amer. J. Anat. 148, 85-120

12. Okamoto N., Satow Y., Lee J. Y., Sumida H., Hayakawa K., Ohdo S. and Okishima T. (1984) Morphology and pathogenesis of the cardiovascular anomalies induced by bis-(dichloroacetyl) diamine in rats. In Congenital Heart Disease: Causes and Processes (ed. Nora J. J. and TaKao A.) Futura Publishing Co., Mount Kisco, pp. 199-221

13. Okishima T., Ohdo S., Hayakawa K. and Okamoto N. (1985) Pathogenesis of a bisdiamine-induced malformation complex in rat resembling DiGeorge syndrome. Cong. Anom. 25, 29-44

14. Sumida H., Nakamura H. and Satow Y. (1990) Distribution of vitronectin in the embryonic chick heart during endocardial cell migration. Arch. Histol. Cytol. 53, 81-88

15. Sumida H., NaKamura H. and Yasuda M. (1992) Role of vitronectin in embryonic rat endocardial cell migration in vitro. Cell Tissue Res. 268, 41-49

16. Taleporos P., Salgo M. P. and Oster G. (1978) Teratogenic action of a Bis(dichloroacetyl)diamine on rats: Patterns of malformations produced in high incidence at timelimited periods of development. Teratology 18, 5-16

17. Yatohgo T., Izumi M., Kashiwagi H. and Hayashi M. (1988) Novel purification of vitronectin from human plasma by heparin affinity chromatography, Cell Struct. Funct. 13, 281-292 